\title{
American Board of Family Medicine (ABFM) Launches New Exam Prep iPhone Application
}

The American Board of Family Medicine (ABFM) is pleased to announce the launch of its new iPhone application: ABFM Examination Prep. This mobile application provides family physicians with useful information that will assist with preparation for the ABFM Maintenance of Certification Examination. This module turns the iPhone into an invaluable tool for keeping in touch with what physicians need to know as they enter their certification or recertification year. The iPhone application is available as a free download from iTunes.

\section{Features}

Features of the ABFM Examination Prep application include:

- Examination prep documents and videos-these include the detailed "ABFM Guide for Examination Preparation" document

- Upcoming examination dates-a quick view of future dates

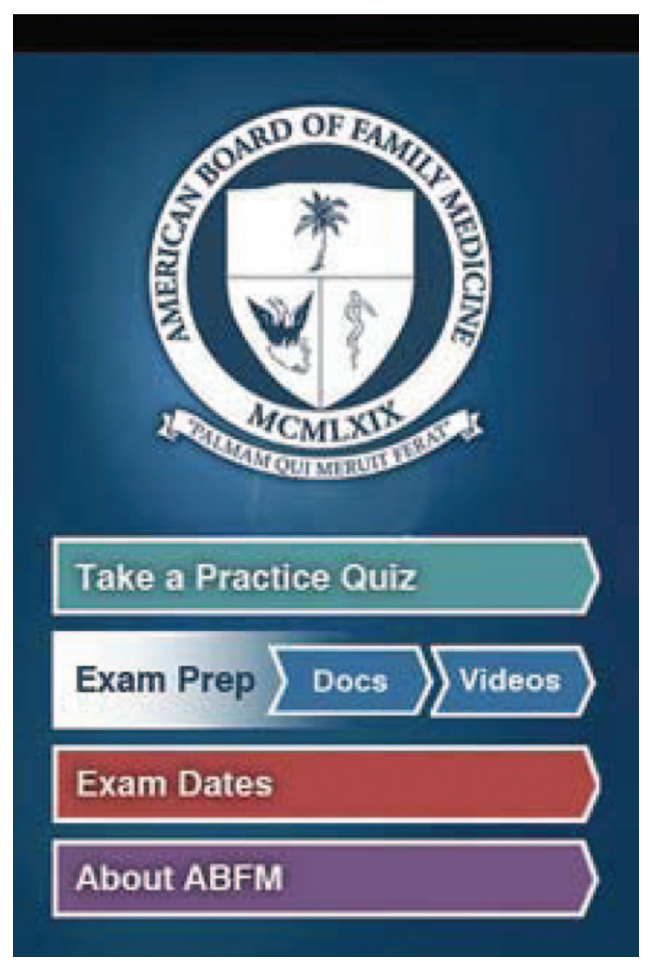

- Links to the ABFM and the Fournal of the American Board of Family Medicine websites

- Practice Quiz-includes more than 200 questions addressing problems commonly encountered by family physicians; each practice quiz presents 10 questions followed by a clear rationale for the correct answer, including the associated reference

Though not a substitute for a well-designed and executed study plan, the ABFM examination preparation application is an additional tool that will help prepare physicians for the Maintenance of Certification Examination. This module is designed specifically for family physicians, but the questions also may be useful for medical students and other health care providers.

For questions regarding the new iPhone application, please contact the ABFM Support Center at 877-223-437 or via E-mail at help@theabfm.org.

Michele Mason

American Board of Family Medicine

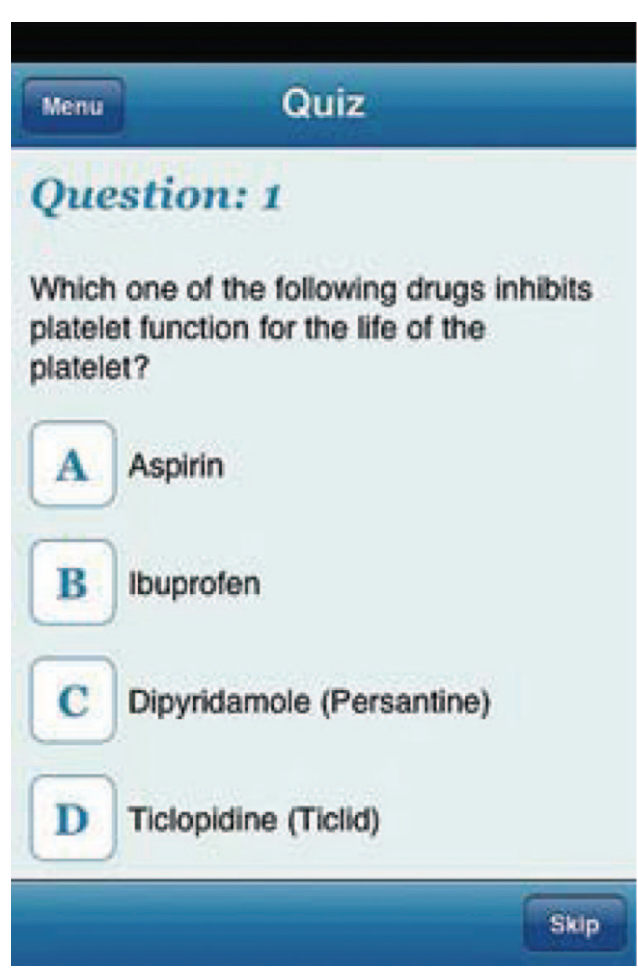

